\title{
Speaking under gum trees with palmtops
}

\author{
Larry Nelson \\ Curtin University of Technology
}

Late in 1989 an article in Australian Personal Computer magazine detailed the imminent release of an all solid-state laptop computer which would run for hours on ordinary pen-light cells (AA batteries).

The computer, a PSION MC-400, was to support digital audio, and, to this end, came with a microphone and speaker built in. The digital audio unit itself was to be made available at a later date, and would be designed to install in the MC-400 much as today's PCMCIA cards slip into current notepad computers. It was claimed that the unit would allow up to eight minutes of audio to be compressed and stored in 64 kilobytes of memory.

I bought an MC-400 as part of a research project looking into the use of computers in vocabulary building, and accepted delivery of the unit, learning how to program its structured BASIC language, "OPL", whilst waiting for its audio digitising plug-in to arrive.

It never did. I consequently abandoned the MC-400 in favour of an Amiga, and then, when Microsoft released Windows 3.0, got support from the DEET ILOTES [1] Project to develop my vocabulary-building and pronunciation-practice exercises on a stock 386-level machine with a sound card (see Nelson, 1993).

Using Visual Basic, I was able to produce prototype lessons in three languages, with each lesson fitting onto a single 1.44M floppy diskette. The lessons featured a set of fixed vocabulary items, ranging from lessons with 40 single words, to lessons with 20 simple phrases. Audio files in Window's WAV format came on each diskette; these featured the voices of female and male native speakers. Lesson users were challenged to learn the vocabulary items via one of three game modes, and could compare their own pronunciation with that of the native speakers, if 
they so wanted, by using record and playback buttons built into the software.

The "DEET Talk" ten Indonesian prototype lessons have proved to be popular with a variety of Western Australian users, including a few large senior high schools. DEET have given permission for the lessons to be freely copied, and some high school students have taken copies home.

\section{Speaking under gum trees}

At the Second International Interactive Multimedia Symposium, I presented a paper (Nelson, 1994) in which I asked readers to consider two possible scenarios: sitting under a gum tree with a tape recorder and set of drill and practice vocabulary flip cards, and sitting under the same gum tree with a multimedia notepad computer running DEET Talk lessons modelled on the same flip cards and audio tape clips.

I asked readers to put costs aside, and to consider what I saw as the relative advantages of the computer, namely, the ability to integrate print and tape media in a package which was, above all, easier to use, more colourful, and potentially much more challenging.

There was no time in my presentation for rebuttal. No one mentioned that it might be seen as folly to think that a notepad's battery would last long enough to make the scenario a practical one; no one cited the matter of component weight and convenience. Even had there been time for discussion, it is unlikely that delegates at a multimedia conference would be found to suggest that old media would come up trumps in any such comparison.

\section{Palmtops}

PSION Computers added digital audio capabilities to their Series 3 palmtop computers in 1995 as part of the upgrade to what is now known as the Series 3a. Acorn Computers have their own version of the Series 3a, called the Pocket Book II.

The 1995 Australian Computers in Education Conference featured demonstrations of the Acorn Pocket Book, and papers were published in the conference proceedings relating to the use of the small Acorns in schools (see, for example, Schibeci and Kissane, 1995). 
For those readers unfamiliar with palmtops, they are essentially fullfledged computers in a package considerably smaller than a VHS video cassette tape. Of necessity their screens and keyboards (if they have one) are small, but their applications software can be extensive, and most operate on AA batteries. Included in the palmtop category are machines such as the Apple Newton MessagePad, the Casio Z-7000 (also known as the Zoomer, Tandy Z-PDA, and the AST GRIDPad 2390), the Hewlett Packard 200LX and OmniGo 100 Organiser Plus, the Motorola Envoy Communicator, and the Sharp ZR-5000 and ZR-5000FX.

The PSION and Acorn palmtops are, to my knowledge, the only ones which incorporate in-built digital audio capabilities. Many of the palmtops support PCMCIA cards; the PSION and Acorn models use solid-state disks, commonly referred to as flash ROMs and RAMs. All palmtops claim to run tens of hours with AA batteries. The Apple Newton, for example, claims to run up to 22 hours on a set of four AAs, while, at the other extreme, Casio say their Z-7000 will deliver 90 hours from three AA cells.

I bought an Acorn Pocket Book II early in 1996, as part of my continuing interest in exploring the use of computer technology in vocabulary development. I have found that two AA Alkaline batteries will power the unit for anywhere from 38 to 62 hours of on time; I have yet to reach the manufacturer's suggested 80 hours, but, at the same time, I probably use digital audio more than the average user. Thus far I have inserted a pair of new batteries every five weeks.

The Acorn's screen is 480 pixels wide, by 160 high. It uses MS DOS as its operating system, and runs a 16-bit NEC V30H CPU at 7.68MHz. Each screen pixel is fully addressable; the graphics capabilities of the computer are comprehensive, although the display is limited to a blackgrey-white plane. The audio system is based on an 8-bit 11-kHz format, and uses the CCITT A-law compression system. A small microphone and speaker are built in. PSION has made available a free utility which will convert Windows WAV files.

Available accessories include Mac-link and PC-link cables, with companion software which makes it a straightforward matter to exchange files, at least between the palmtop and other computers which use an MS DOS file structure. 
Standard on-board memory for the PSION 3a and Acorn Pocket Book II is now either $1 \mathrm{MB}$ or $2 \mathrm{MB}$. A $1 \mathrm{MB}$ Acorn cost Aus $\$ 700$ ex-tax in April, 1996; the price of the PSION has been below the Acorn, but the Acorn comes with a slightly-enhanced suite of applications, including a graph plotter. Applications are housed in system ROM, which, in the case of the Acorn, is $2 \mathrm{MB}$.

The standard programming language with the PSION (and Acorn) is still OPL, as on the MC-400, but it's now graphics capable; $C$ compilers are available. It is possible to divide up the PSION's screen into as many as eight separate text and or graphics windows; programming flexibility is considerable. The QWERTY keyboard has separate menu and help keys.

\section{DEET Talk is now TalkAuto}

My initial experiments with palmtop audio on the PSION/Acorn platformette have involved content from the same vocabulary lessons seen in the DEET Talk prototypes, and I have used the same audio files, converting them to the "WVE" format required via the conversion utility mentioned above.

As capable as the palmtop is, event-driven programming based on mouse movements and clicks is out. The PSION MC-400 had a touch panel which supported mouse-like, point and click actions, but the PSION palmtop has nothing similar. I have had to change the lesson modes substantially.

The spirit of the initial TalkAuto lessons is pictured in the screen capture shown below. The vocabulary word or phrase to be mastered is displayed on the first line, followed by its translation in the "other" language. The first line displays for anywhere from zero to 20 seconds before the second line appears. The second line lingers on the screen for anywhere from zero to 20 seconds before the screen is cleared and the next item presented. When the last item has displayed the process begins again, sounding a soft beep as it does to point out to the user that he or she has just completed a cycle through all the items in the lesson, and is embarking on a new cycle.

When the "Play sound" setting is on, the word or phrase is played through the computer's speaker immediately before the second line comes on, and then again immediately before it goes off. The user can 
control some of the action of the program through a menu of seven settings, as shown in the screen image below.

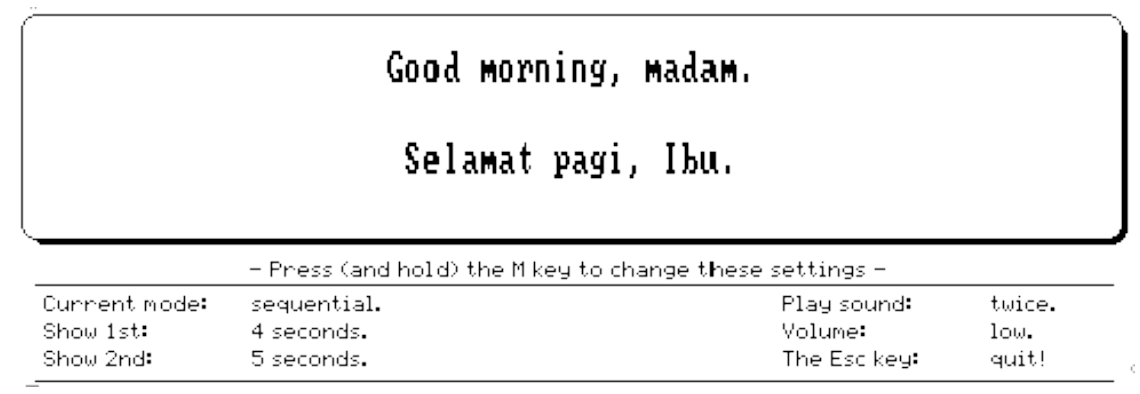

The mode setting determines if the items will be displayed in a sequential manner, or in random fashion. The third setting defines which language appears first, English or the "other" language. Speak may be set at never, once, or twice. When set at once the sound is played only after the second line has been displayed for the number of seconds set on the "Show $2^{\text {nd" }}$ setting.

\begin{tabular}{|c|c|}
\hline \multicolumn{2}{|c|}{ Settings } \\
\hline Modes & -Randorn + \\
\hline Show 1st (secs.): & 4 \\
\hline 'Language 1st: & English \\
\hline 'Show 2nd (secs.): & 5 \\
\hline Speak: & Twice \\
\hline - Uolume leuel: & Medium \\
\hline 'Card set: Name & Gday1 \\
\hline Disk & $\hat{A}$ \\
\hline
\end{tabular}

Lessons are conveniently selected via the "Card set: Name" line, which cycles through available lessons as the user presses the keyboard's right or left arrow keys.

The "Disk" line points to the location of the lessons, which may be on a solid-state disk in one of two drive bays, or on the internal RAM disk. For that matter, if the palmtop is linked to another MS DOS computer, the lessons may be run from a second machine. 


\section{Lesson creation}

TalkAuto lessons presently incorporate two types of data file. The words and phrases are kept in a simple card file, one card per word or phrase. The PSION's "Cards" application makes development of these cards easy. A Windows version of the Cards applet is available from PSION, making it possible to type lesson material on a computer with a larger keyboard.

The other type of file is an audio file. Ideally, there will be one PSION "WVE" file for each card; if a card does not have a corresponding WVE file then nothing is heard as the card's contents are displayed on the screen.

In theory the words and phrases corresponding to the WVE files can be spoken directly into the PSION's microphone. Recording makes heavy demands on the batteries, but if one uses a power adaptor, serious audio recording and editing can be attempted on the palmtop itself; there is a very good freeware wave editor for the PSION on the Internet. However, I do my recordings with a tape recorder, making digital audio clips from the tape by using the Windows Sound System, a Microsoft product.

As TalkAuto evolves, I expect BMP or GIF images will come to be incorporated in some lessons. The standard graphics format on the PSION is "PIC"; converters are readily available to take BMP and GIF files into PIC format.

\section{Adults only}

The TalkAuto lessons are drill and practice with little dressing. Their DEET Talk ancestors are much more animated, having a popular game mode which lets users track their ability to correctly identify a foreign word or phrase within a pre-set number of seconds.

I think it unlikely that younger users would find sufficient challenge in TalkAuto, and am considering ways of making some sort of game which keeps score. The computer might, for example, randomly select one of the words or phrases, play the corresponding sound through the speaker, and wait so many seconds for the user to identify the item by correctly finding it in a list of text or graphics items placed on screen. The items could have a single digit next to them, and the user would signal her or his choice by a press on one of the digit keys. 
A game mode such as this is well within the capabilities of the OPL programming language.

\section{Gum trees with palmtops?}

Let's take the palmtop and revisit my favourite gum tree. Two years ago passing students saw me sitting under the tree, engaged in using flip cards and a tape recorder one day, and a Windows audio-equipped laptop running DEET Talk lessons the next. This year the flip cards and tapes are once again on hand, but the laptop has been replaced by an Acorn palmtop.

We'll work through the first dialogue found in L Fredriksson's Functional Indonesian short course (Fredriksson, 1987). The cards and tape are familiar; the audio quality on the tape is excellent. The problem with these media is that they are not designed to be used conjunctively. The flip cards, by themselves, are fine, but they give no clue to pronunciation. The tape is superb at revealing pronunciation nuances, but there's no corresponding print image of the words heard coming through the headphones.

If I want to have the tape play the last word or phrase again I have to mess with the rewind and play keys, and I always overshoot the mark, rewinding too far. This stage would seem set for a new character. Enter stage left multimedia technology.

Two years ago the Windows laptop did a good job in its cameo role as a new star. But it was a bit bulky, very expensive, and not good for a performance lasting more than a couple of hours. Nonetheless, its audio quality was good, as was its visual display. It would be difficult to use under more cramped conditions such as a coach seat on an airplane, bus, or train, but in the great outdoors elbow room was not an issue.

This year the palmtop provides the multimedia.

As a replacement for flip cards it is excellent. The computer's visual display is small, but even so it's larger than flip cards, with just as much if not more contrast. As a replacement for the tape recorder, however, it suffers when compared to the Windows laptop. The audio quality is similar to that of the smallest of transistor AM radios, and the volume range is not extensive at all. If many students are passing close by, or if the wind is stirring the branches above, the palmtop's speaker has to be 
put right next to one's ear. In this position the audio comes through well, but having the computer at the side of the head makes it rather difficult to read its screen. An earphone jack would solve this problem.

What the palmtop has in its favour is portability. If we were to think of having hundreds of flip cards, just flip cards alone, the palmtop would be a better way to carry them about. In a quiet setting the palmtop can replace the tape recorder; the convenience of having two media, print and tape, well coordinated in a small package, has real attraction. And, unlike the Windows laptop, the palmtop will last out tens of hours before requiring a battery fix.

Have I, then, found a truly portable, use-anywhere replacement for flip cards and cassette tapes, a functional multimedia marriage of two old incompatible media? Not quite.

\section{Limitations}

A palmtop such as that used in the work I have described has two drawbacks, one minor and one major.

The minor one relates to the lack of audio volume mentioned above. This would be easy to fix - equip the unit with an earphone jack.

The major problem relates to file storage. The PSION and Acorn computers use all solid-state memory devices, and such memory is expensive. In July 1990, a 512KB solid-state flash (EPROM) disk cost Aus $\$ 425$, while its more flexible battery-backed solid-state RAM cousin cost Aus\$650. In 1996 prices for these devices had fallen by about half, but even so the purchase of half a megabyte of disk storage still required an outlay of hundreds of dollars. This situation is ameliorated somewhat by falling costs for CMOS RAM, which the PSION and Acorn use for core memory. A PSION or Acorn palmtop with two megabytes of user CMOS RAM became available just after I purchased a one megabyte model (a familiar story to many of us, no doubt), and the price of the extra megabyte of central memory was on the order of Aus $\$ 100$.

In terms of the audio work I have done in recent years, $512 \mathrm{~KB}$ is sufficient to host about 80 digitised words in the 8 -bit, $11 \mathrm{kHz}$ PSION format. Fredriksson's initial dialogue mentioned above involves eighteen phrases, requiring $287 \mathrm{~K}$ of storage on the Acorn palmtop. 
A factor or relevance here is that PSION have file managers which make it easy to move subdirectories, or blocks of files, over a serial cable. This makes it possible to set up lesson-images on an MS DOS hard disk and download them when required to the Acorn.

\section{A spin-off}

My work in the palmtop world has sparked a new appreciation of the KISS [2] design philosophy. I stopped developing audio lessons of the DEET Talk sort when central funding dried up. DEET Talk lessons are made to appeal to today's multimedia audience, using colourful graphics, pop-down menus, and other such common baubles. Although the assembly of such lessons is a straightforward matter, the required work is tedious and time consuming.

In point of fact, an adult learner is likely to be just as challenged by the simple, no-frills TalkAuto lessons now running on the palmtop. Such lessons can be put together literally in a manner of minutes, or, say, no more than two hours, assuming the needed audio clips have already been catalogued. Accordingly I now sense the advantage of coming back to an audio-equipped notepad platform with a refreshed outlook on lesson design - a simpler lesson style will make it possible to create material at minimal cost, and in much less time, but will, I suspect, still get the job done in a satisfactory manner.

\section{Summary}

There would perhaps be few cases where the advantage of multimedia over traditional media is more apparent than in language learning. Over the years, I have investigated the feasibility of developing and delivering drill and practice vocabulary and pronunciation exercises on a variety of microcomputers, including the Apple II, the PSION MC-400, an Amiga 2000, 386-level Windows machines, and now on audio-ready, MS DOScompatible palmtops.

To my mind the palmtop is the winning platform due to its excellent portability. Unfortunately the cost of solid-state memory is presently prohibitive. As time passes memory prices will decline, while enhanced audio compression techniques will make it possible to squeeze more sound into less memory. It does not seem unrealistic to think that small, all solid state computers will provide viable language learning platforms in the near future. 


\section{Addendum}

The palmtop field has seen some salient developments in the 6 months since the development of this paper. Microsoft's Windows CE (Consumer Electronics) operating system has emerged as the operating system of choice for many vendors of new palmtops. The first round of WinCEequipped handhelds supported the playback of WAV files; in this round, one machine, the Philips Velo 1, supported WAV file recording as well as playback. I have experimented with the Velo 1, and found its audio playback quality to be no better than that found on the PSION / Acorn mentioned in this paper. Meanwhile, PSION have substantially upgraded their palmtop; the new version has a pen-based graphical user interface.

Microsoft have released a version of Visual Basic which allows systems to be developed on a desktop platform and ported to a WinCE device. Prices have dropped. As this paper went to print, one could buy a palmtop with $8 \mathrm{MB}$ of RAM for less than Aus $\$ 500$. This price would be for a first-round WinCE computer; prices for second-round machines neighbour on Aus\$800, with new full-colour models selling for over Aus $\$ 1,200$.

Copies of the PSION / Acorn software cited in this paper may be downloaded from http: / ass.curtin.edu.au/ systems /

\section{Notes}

1 Australian Department of Employment, Education and Training. Innovative Languages Other than English in Schools (1992).

2 Keep it simple, stupid.

\section{References}

Fredriksson, L (1987). Functional Indonesian, a short conversational course. Fredriksson Associates: Nedlands, Western Australia.

Nelson, L.R. (1993). Creating and using digital audio files under the Windows operating environment. Australian Journal of Educational Technology, 9 (1), 1-11. http:/ / www.ascilite.org.au/ajet/ ajet9/ nelson.html

Nelson, L.R. (1994). A working multimedia equivalent to Jurassic languagelearning flip cards and tapes. In Proceedings of the 2 nd International Interactive Multimedia Symposium, 364-370, Perth: Promaco Conventions Pty Ltd. http: / / www.ascilite.org.au/aset-archives/confs/iims/1994/np/nelson.html

Schibeci, R. \& Kissane, B. (1995). Learning with palmtop computers. In Learning without limits, proceedings of the Australian Computers in Education Conference 1995, 91-98, Perth: Educational Computing Association of Western Australia. 